\title{
STATISTICAL PROPERTIES OF A HETEROGENEOUS ASSET PRICING MODEL WITH TIME-VARYING SECOND MOMENT
}

\author{
CARL CHIARELLA* ${ }^{*}$ XUE-ZHONG HE* AND DUO WANG ${ }^{\dagger}$ \\ ${ }^{*}$ School of Finance and Economics \\ University of Technology, Sydney \\ PO Box 123 Broadway \\ NSW 2007, Australia \\ ${ }^{\dagger}$ LMAM \\ Department of Financial Mathematics \\ School of Mathematical Sciences \\ Peking University \\ Beijing 100871 \\ People's Republic of China
}

\begin{abstract}
Stability and bifurcation analysis of deterministic systems has been widely used in modeling financial markets. However, the impact of such dynamic phenomena on various statistical properties of the corresponding stochastic model, including skewness and excess kurtosis, various autocorrelation (AC) patterns of under and over reactions, and volatility clustering characterised by the long-range dependence of ACs, is not clear and has been very little studied. This paper aims to contribute to this issue. Through a simple behavioural asset pricing model with fundamentalists and chartists, we examine the statistical properties of the model and their connection to the dynamics of the underlying deterministic model. In particular, our analysis leads to some insights into various mechanisms that may generate some of the stylised facts, such as fat tails, skewness, high kurtosis and long memory, observed in high frequency financial data.
\end{abstract}

JEL classifications: D83; D84; E21; E32, C60

Keywords: Fundamentalists, chartists, stability, bifurcation, investors' under- and over-reactions, stylized facts.

\section{INTRODUCTION}

As a result of a growing dissatisfaction with (i) models of asset price dynamics based on the representative agent paradigm, as expressed for example by Kirman (1992), and (ii) the extreme informational assumptions of rational expectations, research into the dynamics of financial asset prices resulting from the interaction of heterogeneous

Financial support for Duo Wang by NSFC.10271007 and RFDP.20010001042 is acknowledged. This paper was prepared when Wang was visiting the University of Technology, Sydney, whose hospitality is gratefully acknowledged. 
agents has developed, including for example Arthur et al. (1997), Brock and Hommes (1997a, 1997b), Brock and LeBaron (1996), Bullard and Duffy (1999), Chen and Yeh (1997, 2002), Chiarella (1992), Chiarella et al. (2002), Chiarella and He (2001, 2002, 2003), Dacorogna et al. (1995), Day and Huang (1990), Farmer and Joshi (2002), Gaunersdorfer (2000), Gaunersdorfer et al (2003), Hommes (2001, 2002), LeBaron et al. (1999), Lux (1995, 1997, 1998) and Lux and Marchesi (1999). In particular, Brock and Hommes $(1997 b, 1998)$ have introduced the concept of an adaptively rational equilibrium, where agents adapt their beliefs over time by choosing from different predictors or expectations functions, based upon their fitness functions measured by realized profits. The resulting dynamical system is capable of generating the entire zoo of complex behaviour from local stability to high order cycles and chaos as various key parameters of the model change. Brock and Hommes's framework has been extended further in Gaunersdorfer (2000) and Chiarella and He (2001, 2002, 2003) to incorporate heterogeneous variance, risk and learning under both Walrasian auctioneer and market maker scenarios. It is found that the relative risk attitudes, different learning mechanisms and different market clearing scenarios affect asset price dynamics in a very complicated way. It has been shown (e.g. Hommes (2002)) that such simple nonlinear adaptive models are capable of explaining important stylized facts, including fat tails, clustered volatility and long memory, of real financial time series.

There are two goals this literature is trying to achieve, first to explain various market behaviour and, second to replicate the econometric properties and stylized facts of financial time series. These heterogeneous agent models, in particular the theoretically oriented ones, have shown great potential in achieving the first goal. However, we still have some distance in achieving the second goal. On the one hand, it is well known that most of the stylized facts can only be observed for high frequency data, such as weekly, daily or intraday data. However, most of the heterogeneous agent models have difficulty in replicating realistic time series at high frequency. On the other hand, stability and bifurcation analysis have been widely accepted tools in investigating such models, but it is not clear yet how various types of bifurcations of the deterministic system can be used to characterize various price volatility and time series properties (such as distribution density and autocorrelations of returns) of the corresponding stochastic asset pricing models. These issues have been tackled recently in He (2003) through a simple fixed fraction heterogeneous asset pricing model. This paper is largely motivated by these issues, in particular by $\mathrm{He}$ (2003), and seeks to contribute to both of the aforementioned goals.

Gaunersdorfer et al. (2003) consider a simple asset pricing model of fundamentalists and chartists. The fundamentalists believe that tomorrow's price will move in the direction of the fundamental price, while chartists derive their beliefs from a simple technical trading rule using only the latest observed price and extrapolation of the latest observed price change. By assuming a constant conditional variance for both types of traders and imposing a penalty function in the fitness function of the chartists (to ensure that speculative bubbles cannot last forever), they show that volatility clustering can be characterized by a coexistence of a stable steady state and a stable limit cycle, which arises as a consequence of a so-called Chenciner bifurcation. Simple economic intuition of this result is also provided. This paper is a significant contribution in relating asset price volatility to the bifurcation nature of the underlying deterministic system. However, it is not clear if such a characterization of volatility clustering still 
holds for the corresponding stochastic system when the fundamental price follows a random process, in particular when the trading frequency is high.

Given the variety of technical trading rules and differing risk aversion of various investors, this paper introduces a risk adjusted demand function for the chartists by assuming that they use a weighted process of past prices to estimate both conditional mean and variance. Therefore their demand function is a nonlinear function of the conditional mean and variance, instead of a linear function of the conditional mean only as in Gaunersdorfer et al. (2003). It is found that the mechanism of variance adjusted demand function of the chartists provides a natural way to prevent the price from getting stuck in a speculative bubble ${ }^{1}$. Similar to Brock and Hommes $(1997 b, 1998)$, an adaptive model based on the fitness function is then obtained. We examine how the price dynamics of the risky asset is affected by the reactions of investors, the switching intensity of the fitness function, and the weighting process and risk adjustment of the chartists.

The plan of the paper is as follows. We first develop a simple adaptive asset pricing model of fundamentalists, chartists and noise traders. A statistical analysis is then conducted when parameters are near the bifurcation boundaries of the underlying deterministic model and a connection between the Hopf (flip) bifurcation and under(over)-reaction AC patterns is established. The paper finishes with a discussion on the capability of the model to generate the stylised facts and the long memory of high frequency financial time series.

\section{THE MODEL}

Following the framework of Brock and Hommes (1998), consider an asset pricing model with one risky asset and one risk free asset. It is assumed that the risk free asset is perfectly elastically supplied at the risk-free rate $r$ (per annum). Let $p_{t}$ be the price (ex dividend) per share of the risky asset at time $t$ and $\left\{y_{t}\right\}$ be the stochastic dividend process of the risky asset. Then the wealth of investor $h$ at $t+1$ is given by

$$
W_{h, t+1}=R W_{h, t}+R_{t+1} z_{h, t}
$$

where

$$
R_{t+1}=p_{t+1}+y_{t+1}-R p_{t}
$$

is the excess capital gain/loss, $R=1+r / K, K$ is the trading frequency per annum ${ }^{2}$, $W_{h, t}$ is the wealth at time $t$ and $z_{h, t}$ is the number of shares of the risky asset purchased at $t$. Denote by $F_{t}=\left\{p_{t-1}, \cdots ; y_{t-1}, \cdots\right\}$ the information set formed at time $t$. Let $E_{h, t}, V_{h, t}$ be the beliefs of investor type $h$ about the conditional expectation and variance, based on $F_{t}$. Then it follows from (2.1) and (2.2) that

$$
E_{h, t}\left(W_{t+1}\right)=R W_{t}+E_{h, t}\left(R_{t+1}\right) z_{h, t}, \quad V_{h, t}\left(W_{t+1}\right)=z_{h, t}^{2} V_{h, t}\left(R_{t+1}\right) .
$$

Assume investor has a CARA (constant absolute risk aversion) utility function $u(W)=$ $-e^{-a_{h} W}$ with risk aversion coefficient $a_{h}>0$, and maximises his/her expected utility

\footnotetext{
${ }^{1}$ In Gaunersdorfer et al. (2003), this is ensured by artificially adding a penalty function in the fitness function of the chartists, which is unnecessary in our model.

${ }^{2}$ Typically, annually, quarterly, monthly, weekly and daily trading periods correspond to $K=$ $1,4,12,52$ and 250 , respectively.
} 
of wealth, leading the optimal demand

$$
z_{h, t}=\frac{E_{h, t}\left(R_{t+1}\right)}{a_{h} V_{h, t}\left(R_{t+1}\right)} .
$$

In the following discussion, we adopt the popular fundamentalist/chartist model by assuming that all investors can be grouped into either fundamentalists (type 1) or chartists (type 2).

Fundamentalists-The fundamentalists are assumed to believe that the expected market price $p_{t}$ is mean reverting to their perceived fundamental value $p_{t}^{*}$ and the conditional variance of the market price is constant. That is,

$$
\left\{\begin{array}{l}
E_{1, t}\left(p_{t+1}\right)=p_{t-1}^{*}+v\left(p_{t-1}-p_{t-1}^{*}\right), \quad 0 \leq v \leq 1 \\
V_{1, t}\left(p_{t+1}\right)=\sigma_{1}^{2}
\end{array}\right.
$$

where $p_{t}^{*}$ is the fundamental price of the risky asset estimated by the fundamentalists at some cost, $v$ is the speed of mean reversion estimated by the fundamentalists, and $\sigma_{1}>0$ is a constant. In particular, $E_{1, t}\left(p_{t+1}\right)=p_{t-1}^{*}$ for $v=0$ and $E_{1, t}\left(p_{t+1}\right)=$ $p_{t-1}$ for $v=1$. The conditional expectation of the fundamentalists (2.5) can also be written as $E_{1, t}\left(p_{t+1}\right)=(1-v) p_{t-1}^{*}+v p_{t-1}, 0 \leq v \leq 1$, which is a weighted average of the fundamental price and latest price. Hence small (large) values of $v$ indicate that the fundamentalists give more (less) weight to the fundamental price and less (more) weight to the latest price, believing that price moves quickly (slowly) towards its fundamental value $p_{t}^{*}$. For convenience of discussion, we say the fundamentalists over(under)-react (to the market price) when more (less) weight $v$ is given to the market price.

Chartists-Unlike the fundamentalists who are able to obtain a good estimate of the fundamental value, chartists base their trading strategy on signals generated from the costless information contained in recent prices. The signal may be generated by comparing the latest price $p_{t-1}$ with some reference price trends $\tilde{p}_{t-1}$, such as a moving average process. Specifically, the chartists consider the realizations of $\tilde{p}_{t-1}$ as random drawn from some distribution. The distribution can be conditional on past realized values. For simplicity, we assume that $\tilde{p}_{t-1}$ is conditionally distributed on prices $p_{t-2}$ and $p_{t-3}$ with weighting probabilities $w$ and $1-w$, respectively. Then the conditional mean and variance of the trend can be estimated, respectively, as

$$
\left\{\begin{array}{l}
\bar{p}_{t-2} \equiv w p_{t-2}+(1-w) p_{t-3}, \quad 0 \leq w \leq 1 \\
\bar{\sigma}_{t-2}^{2} \equiv w\left[p_{t-2}-\bar{p}_{t-2}\right]^{2}+(1-w)\left[p_{t-3}-\bar{p}_{t-2}\right]^{2} .
\end{array}\right.
$$

Based on the trading signals $p_{t-1}-\tilde{p}_{t-1}$ and the conditional mean and variance estimates (2.6), we make the following assumptions for the chartists:

$$
\left\{\begin{array}{l}
E_{2, t}\left(p_{t+1}\right)=p_{t-1}+g\left(p_{t-1}-\bar{p}_{t-2}\right), \quad g \in \mathbb{R} \\
V_{2, t}\left(p_{t+1}\right)=\sigma_{1}^{2}\left[1+b \bar{\sigma}_{t-2}^{2}\right], \quad b \geq 0
\end{array}\right.
$$

where $g \in \mathbb{R}$ is the estimated extrapolation rate of the chartists. That is, the chartists' beliefs are based on the latest price and their extrapolation of the trading signals generated from the trend. In particular, chartists are called trend followers when $g>0$ and are contrarians when $g<0$. For $w=1, E_{2, t}\left(p_{t+1}\right)=p_{t-1}+g\left(p_{t-1}-p_{t-2}\right)$ which is the case discussed in Gaunersdorfer et al. (2003) and, for $w=0, E_{2, t}\left(p_{t+1}\right)=$ $p_{t-1}+g\left(p_{t-1}-p_{t-3}\right)$. Similarly, for convenience of discussion, we say the chartists over(under)-react when they extrapolate strongly (weakly), that is when $|g|$ is large 
(small). With regard to the chartists' estimate of the variance, they use the historical variance to scale up the fundamental variance through the parameter $b$. High $b$ reflecting a greater sensitivity to variance risk.

For the dividend process, we assume $E\left(y_{t}\right)=\bar{y}, V\left(y_{t}\right)=\sigma_{y}^{2}$. In particular, when $p_{t}^{*}=p^{*}$ is constant, $\bar{y}=r p^{*}$. Based on the above assumptions, the optimal demands for the fundamentalists and chartists are given, respectively, by

$$
\left\{\begin{array}{l}
z_{1, t}=\left[p_{t-1}^{*}+v\left(p_{t-1}-p_{t-1}^{*}\right)+\bar{y}-R p_{t}\right] / A_{1} \\
z_{2, t}=\left[p_{t-1}+g\left(p_{t-1}-\bar{p}_{t-2}\right)+\bar{y}-R p_{t}\right] / A_{2, t}
\end{array}\right.
$$

where

$$
A_{1}=a_{1}\left(\sigma_{1}^{2}+\sigma_{y}^{2}\right), \quad A_{2, t}=a_{2}\left[\sigma_{y}^{2}+\sigma_{1}^{2}\left(1+b \bar{\sigma}_{t-2}^{2}\right)\right]
$$

and $\bar{p}_{t}$ and $\bar{\sigma}_{t}^{2}$ are defined by (2.6).

Let $U_{1, t}$ and $U_{2, t}$ be the realized profit of the fundamentalists and chartists, respectively, defined by $U_{i, t}=R_{t} z_{i, t-1}-C_{i}, i=1,2$, where $C_{i} \geq 0$ measures the total cost incurred by the agents in generating information to form their estimates. Let $n_{i, t}$ be the fractions of agents of type $i$ at time $t$. Assume the fractions are formed on the basis of discrete choice probability (see Manski and McFadden (1981), Anderson, de Palma and Thisse (1993), Brock and Hommes (1997b, 1998)), namely

$$
n_{i, t}=\exp \left[\beta U_{i, t-1}\right] / Z_{t-1}, \quad(i=1,2), \quad Z_{t-1}=\sum_{i=1}^{2} \exp \left[\beta U_{i, t-1}\right],
$$

where $\beta(>0)$ is the intensity of choice measuring how fast agents switch among different prediction strategies. Let $m_{t}=n_{1, t}-n_{2, t}$. Then $n_{1, t}=\left(1+m_{t}\right) / 2, n_{2, t}=$ $\left(1-m_{t}\right) / 2$ and

$$
m_{t}=\tanh \left[\frac{\beta}{2} R_{t-1}\left(z_{1, t-2}-z_{2, t-2}\right)-\frac{\beta}{2} C\right],
$$

where $C=C_{1}-C_{2}$. It is assumed that the fundamentalists expend more on generating relevant information to generate their estimates so that the constant $C \geq 0$ in general.

To clear the market through a Walrasian scenario, we introduce noise traders whose supply of the risky asset is denoted by $z_{n, t} \sim \mathcal{N}\left(0, \sigma_{n}^{2}\right)$. The market is viewed as finding the price $p_{t}$ that equates the sum of these demand from both fundamentalist and chartist schedules to the supply of the noise traders via

$$
\left(1+m_{t}\right) z_{1, t}+\left(1-m_{t}\right) z_{2, t}=z_{n, t} .
$$

Substituting (2.8) and (2.11) into (2.12), one obtains the market cleaning price $p_{t}$

$$
p_{t}=\frac{F\left(p_{t-1}, p_{t-1}^{*}, m_{t}, \bar{p}_{t-2}, \bar{\sigma}_{t-2}^{2}, z_{n, t}, y_{t}\right)}{G\left(p_{t-1}, p_{t-1}^{*}, m_{t}, \bar{p}_{t-2}, \bar{\sigma}_{t-2}^{2}, z_{n, t}, y_{t}\right)}
$$

where

$$
\begin{gathered}
F\left(p_{t-1}, p_{t-1}^{*}, m_{t}, \bar{p}_{t-2}, \bar{\sigma}_{t-2}^{2}, z_{n, t}, y_{t}\right)=A_{2, t}\left[1+m_{t}\right]\left[p_{t-1}^{*}+v\left(p_{t-1}-p_{t-1}^{*}\right)+\bar{y}\right] \\
+A_{1}\left[1-m_{t}\right]\left[p_{t-1}+g\left(p_{t-1}-\bar{p}_{t-2}\right)+\bar{y}\right]-z_{n, t} A_{1} A_{2, t}, \\
G\left(p_{t-1}, p_{t-1}^{*}, m_{t}, \bar{p}_{t-2}, \bar{\sigma}_{t-2}^{2}, z_{n, t}, y_{t}\right)=R\left[\left(1+m_{t}\right) A_{2, t}+A_{1}\left(1-m_{t}\right)\right],
\end{gathered}
$$

$\bar{p}_{t}, A_{1}$ and $A_{2, t}$ are defined by (2.6) and (2.9). Because of the stochastic nature of the dividend process $y_{t}$, the fundamental value $p_{t}^{*}$ and the noise supply, the equilibrium 
price equation (2.13) is equivalent to a high-order nonlinear stochastic discrete system in general.

When the stochastic terms in equation (2.13) take their mean levels, one obtain the corresponding deterministic system. For the deterministic system, it is found from Chiarella et al. (2004) that the constant fundamental price $p^{*}$ is the unique steady state price of the system. In addition, the steady state price becomes unstable through a Hopf bifurcation only when the chartists are trend followers (that is when $g>0$ ). However, when the chartists are contrarians (that is when $g<0$ ), the local stability region is bounded by both Hopf and flip bifurcations. However, depending on the weighting parameter $w$, one of the boundaries may be unbinding. In particular, the steady state price can become unstable through a Hopf bifurcation when $w$ is small, or a flip bifurcation when $w$ is large, or both bifurcations when $w$ is near 0.5 . We refer to Chiarella et al. (2004) for more detailed analysis on the dynamics of the deterministic system. In the following discussion, we examine the statistical properties of the market price of the stochastic model (2.13). In particular we are interested in how statistical properties exhibit different behaviour near different types of bifurcation boundaries of the deterministic system. In so doing, we obtain some insights into the interaction of the dynamics of the deterministic system and the noise processes.

In the following, we choose the fundamental price $p_{o}^{*}=\$ 100$, annual risk-free rate $r=5 \%$, annual volatility of the fundamental price $\sigma=20 \%$. Trading frequency $K=250$, which corresponds to a daily trading period. Correspondingly, the total risk-free return per trading period $R=1+r / K=1.0002$, daily price volatility $\sigma_{1}^{2}=\left(p^{*} \sigma\right)^{2} / K=8 / 5$ and daily dividend volatility $\sigma_{y}^{2}=r^{2} \sigma_{1}^{2}=1 / 250$. We also choose the risk aversion coefficients for both types of investor as $a_{1}=a_{2}=0.8$, the cost difference $C=0$, the coefficient of endogenous variance component $b=1$ and the switch intensity $\beta=0.1$.

We assume that the fundamental price $p_{t}^{*}$ follows a stationary random walk process

$$
p_{t}^{*}=p_{t-1}^{*}\left[1+\epsilon_{t}\right], \quad \epsilon_{t} \sim N\left(0, \sigma_{p}^{2}\right), \quad p_{0}^{*}=\$ 100.00 .
$$

Fig.2.1 displays a realisation of the fundamental prices, $p_{t}^{*}$ and the corresponding relative return $r_{t}=p_{t}^{*} / p_{t-1}^{*}-1$. This fundamental price is used for all of the following simulations. The simulation contains 5,000 observations, corresponding to about 20 years of daily prices. The corresponding statistical properties, including distribution density of the return $r_{t}$ and the autocorrelations (ACs) of $r_{t},\left|r_{t}\right|$ and $r_{t}^{2}$, are also included in Fig. 2.1 One can see that the ACs of $r_{t},\left|r_{t}\right|$ and $r_{t}^{2}$ are not significant at the 95\% level, which is consistent with the stationary random walk process (2.14).

\section{Hopf BifurCATIONS AND UNDER-REACTION AC PATTERNS}

Chiarella et al. (2004) have extensively analysed the dynamical properties of the deterministic skeleton of the price dynamics (2.13). It is found that the fundamental steady state price of the underlying deterministic system becomes unstable through a Hopf bifurcation only in two situations. The first case occurs when the chartists are trend followers (with any weighting parameter $w$ ) and the second case occurs when the chartists are contrarians with lower weighting parameter $w$. We now examine the stochastic properties of the market price when parameters are near the Hopf bifurcation boundary in these two different situations. 

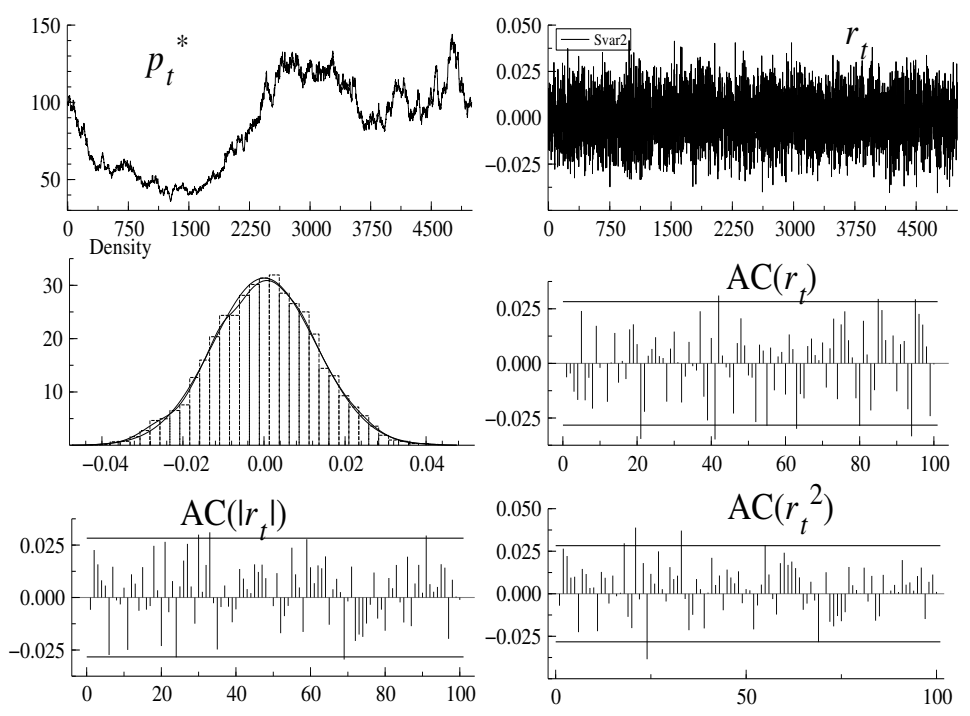

FIGURE 2.1. Time series plots of the prices $p_{t}^{*}$, returns $r_{t}$ and autocorrelations of $r_{t},\left|r_{t}\right|$ and $r_{t}^{2}$.

3.1. Case 1. We first consider the case when the chartists are trend followers. It is found from various simulations that prices become explosive when (i) the parameters $v, g$ and $w$ are either near or outside the Hopf bifurcation boundary, or (ii) the switching intensity $\beta$ is high. Also, an increase in parameter $b$ can make prices become less explosive. Given the destabilizing effect of the trend following strategy, this result is very intuitive. To see the impact of the Hopf bifurcation on the stochastic properties of the stochastic model, we choose $w=0.5, g=1$ and two different values for $v=0.3$ and 0.9. It can be verified that the steady state price of the deterministic model is locally stable with these selections and, in addition, the parameter moves closer to the Hopf bifurcation as $v$ increases. In fact, the corresponding Hopf bifurcation values of $g$ for $v=0.3$ and 0.9 are given by $g^{*}=1.458$ and 1.3 , respectively.

To see the impact of the stochastic fundamental price and noise traders on the price dynamics, we consider two different situations: $\sigma_{n}=0$ and $\sigma_{n}=1$. For $\sigma_{n}=0$, the market price is influenced by the random fundamental price $p_{t}^{*}$ only. When $\sigma_{n}=1$, the market price is influenced by both the random fundamental price $p_{t}^{*}$ and the noisy supply. Fig. 3.1 illustrates the time series of the market price $p_{t}$, the densities of the market fraction $m_{t}$ and the return $r_{t}$, and the ACs of $r_{t},\left|r_{t}\right|$ and $r_{t}^{2}$ for four combinations of $\left(v, \sigma_{n}\right)=(0.3,0),(0.3,1),(0.9,0)$ and $(0.9,1)$. Our numerical simulations show that the features illustrated in Fig. 3.1 are robust, from which we arrive at the following observations.

(i). The market prices $p_{t}$ follow closely the random fundamental price $p_{t}^{*}$ in all cases. This may partially be due to the local stability of the steady state price of the underlying deterministic model, partially due to the mean reverting expectation scheme of the fundamentalists. 

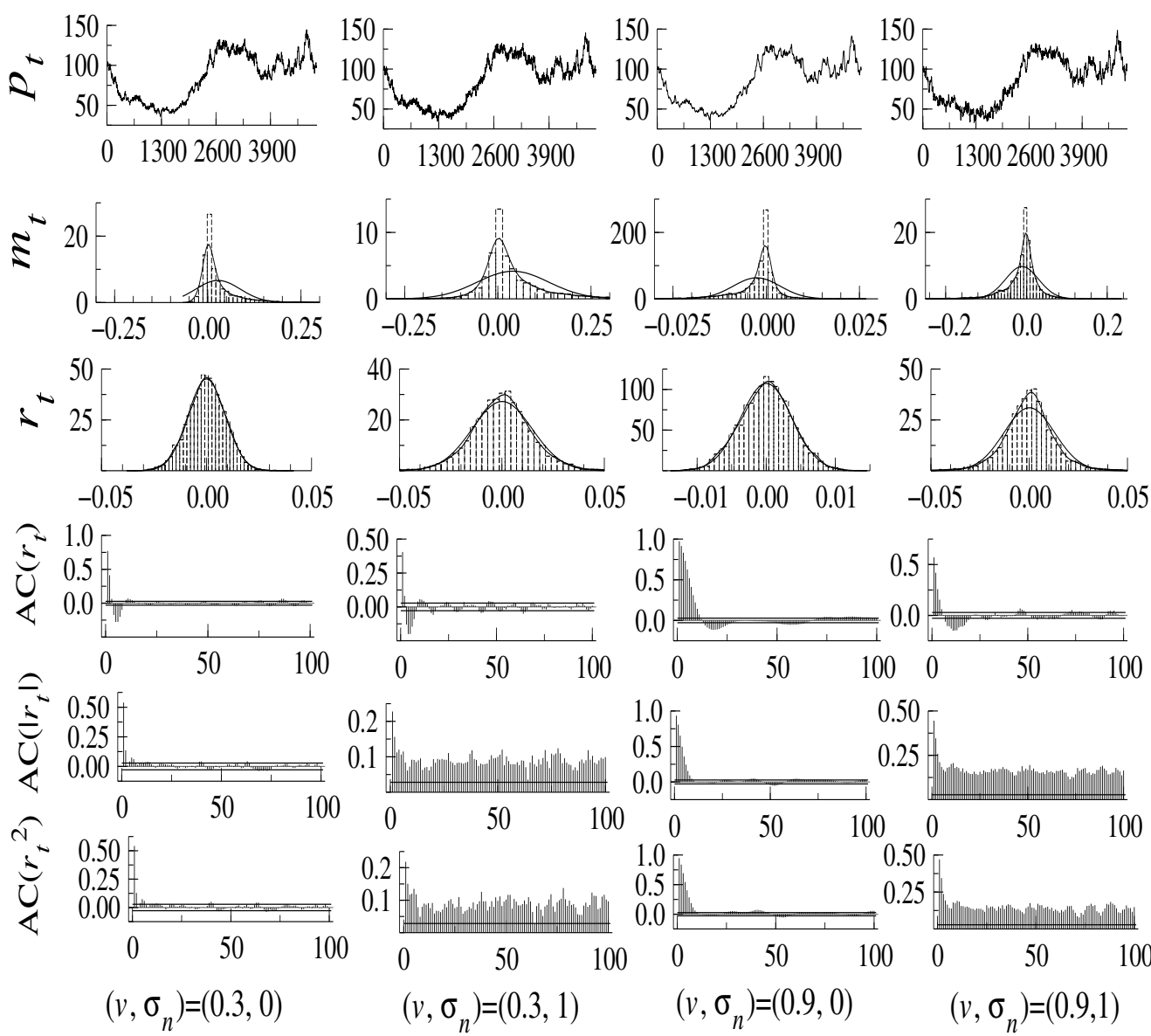

FIGURE 3.1. Time series of the market price $p_{t}$, distribution density of the population fraction difference $m_{t}$ and return $r_{t}$ (compared with the normal distribution) and ACs of $r_{t},\left|r_{t}\right|$ and $r_{t}^{2}$ for $w=0.5, \beta=$ $0.1, a=0.8, g=1, b=1, C=0$, two different values of $v=0.3$ and 0.9 and without, with the noisy supply $\sigma_{n}=0,1$.

(ii). The returns are not normally distributed as indicated by the significant ACs in all cases. However, including noise traders makes the returns even less normal, leading to skewness and high kurtosis.

(iii). The ACs of returns show a strong under-reaction pattern, characterised by the oscillating ACs with positive ACs for small lags, as the parameters move closer to the Hopf bifurcation (the cases $\left(v, \sigma_{n}\right)=(0.3,0)$ and $\left.(0.9,0)\right)$. Such strong patterns are be washed out by introducing the noise traders (the case $\left(v, \sigma_{n}\right)=$ $(0.3,1)$ and $(0.9,1))$.

(iv). The noise traders make a significant contribution to the volatility clustering effect characterised by the significant ACs of the $\left|r_{t}\right|$ and $r_{t}^{2}$ in cases of $\left(v, \sigma_{n}\right)=$ $(0.3,1)$ and $(0.9,1)$. 
(v). The distribution of the population difference $m_{t}$ shows that the investors tend to switch toward the fundamental (trend following) strategy as the parameters move away from (closer to) the Hopf bifurcation boundary, though the volatility of $m_{t}$ is increased by the introduction of the noise traders.

Overall, we can see that the Hopf bifurcation can be used to explain the under-reaction of AC patterns. Also the stylised facts, including skewness, kurtosis, volatility clustering and significant ACs of $\left|r_{t}\right|$ and $r_{t}^{2}$, are observed when the noise traders act as liquidity traders.

3.2. Case 2. We now consider the case when the chartists are contrarians and the weight parameter $w$ is small. In this case, the flip bifurcation boundary becomes nonbinding. It is found from simulations that, different from the previous case, prices do not become explosive on both sides of the Hopf bifurcation. An intuitive explanation is the fact that both fundamental and contrarian strategies are stabilizing strategies. This may also be due to the unbinding flip bifurcation. To see how the market price behavior near the Hopf bifurcation, we choose $v=0.5, w=0.3$ and $g=-1$ and -3 , which are located on both sides of the Hopf bifurcation value $g^{*}=-2.65$ of the underlying deterministic system.

Fig. 3.2 illustrates the time series of the market price $p_{t}$, the densities of the market fraction $m_{t}$ and the return $r_{t}$, and the ACs of $r_{t},\left|r_{t}\right|$ and $r_{t}^{2}$ for four combinations of $\left(g, \sigma_{n}\right)=(-1,0),(-1,1),(-3,0)$ and $(-3,1)$. Similar statistical properties are found when the parameters are located inside the local stability region, but near the Hopf bifurcation boundary, of the underlying deterministic system (that is the cases $\left(g, \sigma_{n}\right)=(-1,0)$ and $\left.(-1,1)\right)$. However, when the parameters are located outside the local stability region, the steady state price of the deterministic system becomes unstable through a Hopf bifurcation and the ACs of returns of the stochastic model display over-reaction patterns characterised by oscillating and decaying ACs which are negative for odd lags and positive for even lags, though the patterns are washed out significantly by the presence of the noise traders.

\section{Flip Bifurcations And Under/Over-Reaction AC Patterns}

This section examines the impact of the flip bifurcation (of the deterministic system) on the stochastic properties of the market price. As we know that the fundamental steady state price of the underlying deterministic system becomes unstable through a flip bifurcation when the chartists are contrarians and the weighting parameter $w$ is higher (in this case the Hopf bifurcation of the underlying deterministic system becomes unbinding). Because of the stablizing effect of both fundamental and contrarian strategies, prices become less explosive on both sides of the flip bifurcation boundary (but more sensitive to the switching intensity $\beta$ ). To see the market price behavior near the flip bifurcation, we choose $v=0.5, w=0.8$ and $g=-1$ and -3 , which are located on both sides of the flip bifurcation value $g^{*}=-2.18773$ of the underlying deterministic system.

Fig. 4.1 illustrates the time series of the market price $p_{t}$, the densities of the market fraction $m_{t}$ and the return $r_{t}$, and the ACs of $r_{t},\left|r_{t}\right|$ and $r_{t}^{2}$ for four combinations of $\left(g, \sigma_{n}\right)=(-1,0),(-1,1),(-3,0)$ and $(-3,1)$. Similar statistical properties are found when the parameters are located inside the local stability region, but near the flip bifurcation boundary, of the underlying deterministic system (that is the cases 

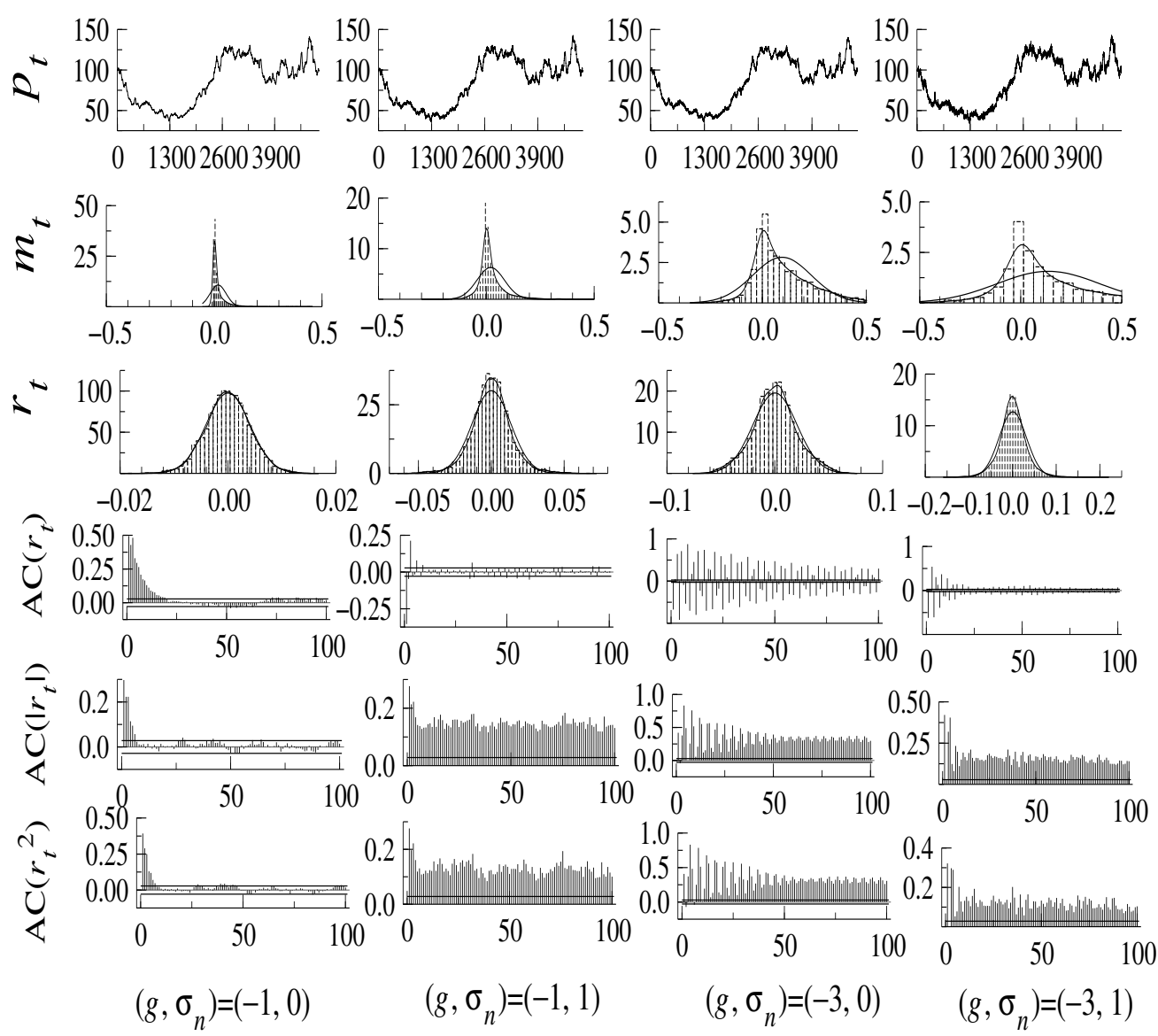

FIGURE 3.2. Time series of the market price $p_{t}$, distribution density of the population fraction difference $m_{t}$ and return $r_{t}$ (compared with the normal distribution) and ACs of $r_{t},\left|r_{t}\right|$ and $r_{t}^{2}$ for $w=0.3, \beta=$ $0.1, a=0.8, v=0.5, b=1, C=0$, two different values of $g=-1$ and -3 and without, with the noise supply $\sigma_{n}=0,1$.

$\left(g, \sigma_{n}\right)=(-1,0)$ and $\left.(-1,1)\right)$. In particular, the under-reaction AC pattern is observed when there are no noise traders. However, for $g=-3$, the steady state price of the underlying deterministic system becomes unstable through a flip bifurcation and both the stochastic fundamental price and the noise traders have different impacts on the stochastic properties of the market price which are summarized as follows:

(vi). Both the population fraction difference $m_{t}$ and the return $r_{t}$ have bi-modal distributions when there are no noise traders present. Correspondingly, the AC displays a very strong over-reaction pattern characterised by slowly decaying oscillating $A C\left(r_{t}\right)$ which are negative for odd lags and positive for even lags. This strong AC pattern is also preserved for the ACs of $\left|r_{t}\right|$ and $r_{t}^{2}$. All these features are underpinned by the flip bifurcation of the underlying deterministic system. 

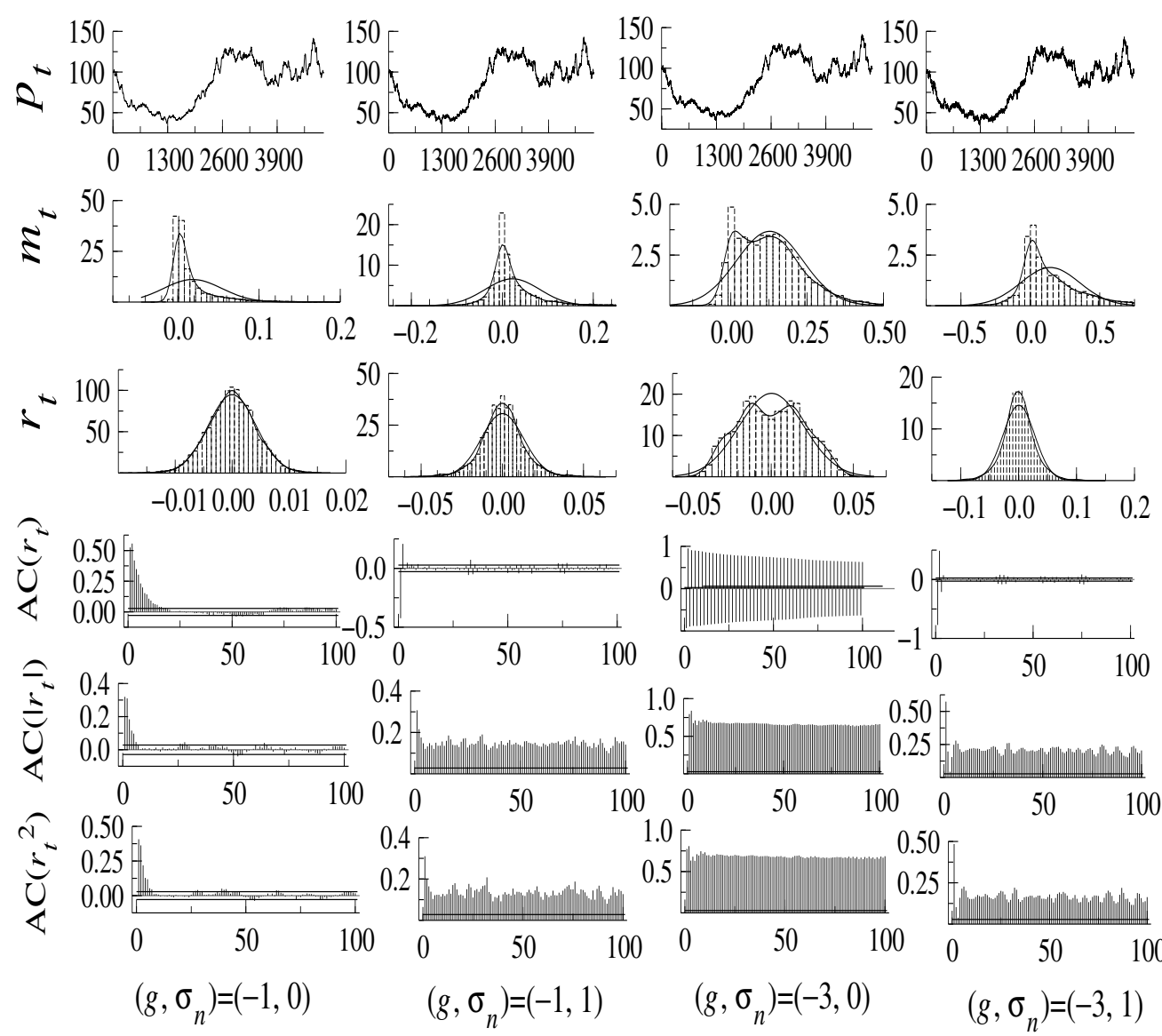

FIGURE 4.1. Time series of the market price $p_{t}$, distribution density of the population fraction difference $m_{t}$ and return $r_{t}$ (compared with the normal distribution) and ACs of $r_{t},\left|r_{t}\right|$ and $r_{t}^{2}$ for $w=0.8, \beta=$ $0.1, a=0.8, v=0.5, b=1, C=0$, two different values of $g=-1$ and -3 and without, with the noisy supply $\sigma_{n}=0,1$.

(vii). When the noise traders are present, most of the statistical features in (vi) disappear. The bi-modal distributions of $m_{t}$ and $r_{t}$ disappear, showing skewness and high kurtosis. In addition, the strong over-reaction AC patterns on returns are washed out and, apart from the first two lags, become insignificant. However, the ACs of both $\left|r_{t}\right|$ and $r_{t}^{2}$ are significant.

Overall, we can see that, when the parameters cross the flip bifurcation of the deterministic system, the ACs pattern of the stochastic returns changes from under-reaction to over-reaction and this is also consistent with trader behaviour. When the noise traders are present, the model generates most of the stylised facts that we discuss in the following section. 


\section{The Stylised Facts and Long Memory}

The above analysis has led us to our first goal-to gain some insights into various types of market price behaviour and to understand the possible mechanism generating such behaviour. We have shown that various statistical aspects of the stochastic system, including distributions and under/over-reaction AC patterns are closely related to the stability and bifurcation analysis of the deterministic system.
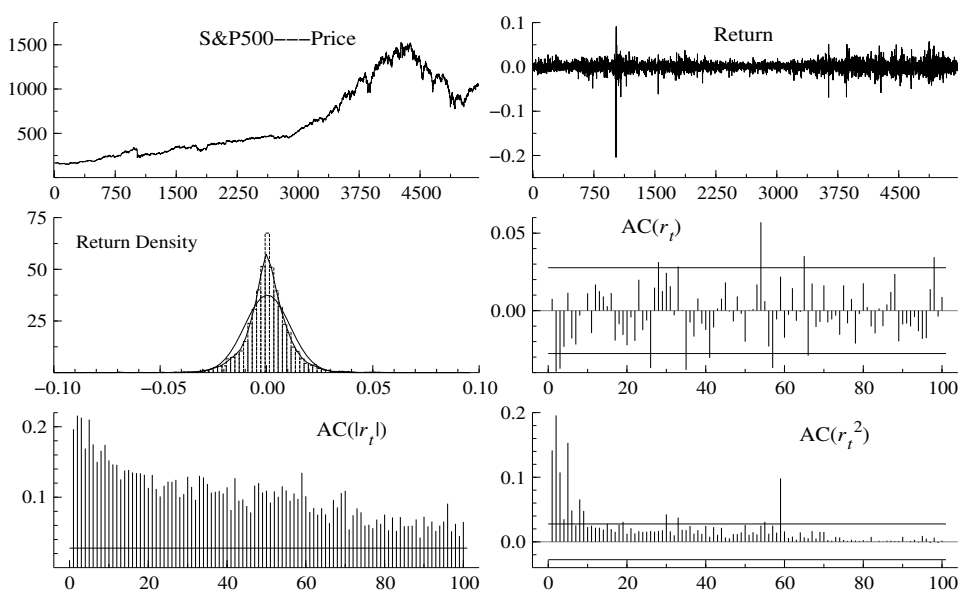

FIGURE 5.1. Time series of the prices and (relative) returns of S\&P500 from 18/11/1983 to 19/11/2003, distribution density of the return (compared with the normal distribution) and ACs of returns, absolute returns and squared returns for lags up to 100 .

We now try to address our second goal, which is the most important and difficult part of asset price modeling, to replicate the econometric properties and the stylized facts of financial time series. As a benchmark of such financial time series, we consider the daily accumulated index of the S\&P500 from 18/11/1983 to 19/11/2003. Fig. 5.1 illustrates the price and (relative) return time series for the last 20 years, the return distribution and the ACs of returns, absolute returns and squared returns for lags up to 100 . For a comprehensive discussion of stylized facts and the so-called long memory of returns of high frequency financial time series, we refer to Ding et al. (1993) and Pagan (1996). The stylised facts include excess volatility, volatility clustering, skewness and excess kurtosis, while the long memory refers to the insignificant ACs on returns, but significant and decayed ACs on the absolute and squared returns, as illustrated by Fig. 5.1 for the S\&P500 daily index. Given the simplicity of our model, it is not easy to reproduce all those features. However, based on our analysis in the previous sections, one can see that the model is indeed able to produce most of those features when the noise traders act as liquidity traders. In particular, for the cases when the chartists are contrarians and the parameters are near either the Hopf or flip bifurcation (but from inside the local stability region) of the underlying deterministic system $\left(w, g, \sigma_{n}\right)=(0.3,-1,1),(0.8,-1,1)$, the return distributions show skewness and excess kurtosis, the distributions of the population fraction difference show some 
herding behaviour, while the ACs of returns display similar patterns to the S\&P500, apart from significant ACs of returns for the first few lags and persistent ACs of the absolute and squared returns.

\section{REFERENCES}

Anderson, S. and A. de Palma and J. Thisse (1993), Discrete Choice Theory of Product Differentiation, MIT Press, Cambridge, MA.

Arthur, W., Holland, J., LeBaron, B., Palmer, R. and Tayler, P. (1997), 'Asset pricing under endogeneous expectations in an artifical stock market', Economic Notes 26(2), 297-330.

Brock, W. and Hommes, C. (1997a), Models of Complexity in Economics and Finance, Wiley, New York, chapter 1, pp. 3-44. in Systems Dynamics in Economic and Finance Models, Eds. Heij, C., Schumacher, J.M., Hanzon, B., Praagman, C.

Brock, W. and Hommes, C. (1997b), 'A rational route to randomness', Econometrica 65, 1059-1095.

Brock, W. and Hommes, C. (1998), 'Heterogeneous beliefs and routes to chaos in a simple asset pricing model', Journal of Economic Dynamics and Control 22, 1235-1274.

Brock, W. and LeBaron, B. (1996), 'A structural model for stock return volatility and trading volume', Review of Economics and Statistics 78, 94-110.

Bullard, J. and Duffy, J. (1999), 'Using Genetic Algorithms to Model the Evolution of Heterogeneous Beliefs', Computational Economics 13, 41-60.

Chen, S.-H. and Yeh, C.-H. (1997), 'Toward a computable approach to the efficient market hypothesis: an application of genetic programming', Journal of Economic Dynamics and Control 21, 10431063.

Chen, S.-H. and Yeh, C.-H. (2002), 'On the emergent properties of artificial stock markets: the efficient market hypothesis and the rational expectations hypothesis', Journal of Economic Behavior and Organization 49, 217-239.

Chiarella, C. (1992), 'The dynamics of speculative behaviour', Annals of Operations Research 37, 101123.

Chiarella, C., Dieci, R. and Gardini, L. (2002), 'Speculative behaviour and complex asset price dynamics', Journal of Economic Behavior and Organization 49, 173-197.

Chiarella, C. and He, X. (2001), 'Asset pricing and wealth dynamics under heterogeneous expectations', Quantitative Finance 1, 509-526.

Chiarella, C. and He, X. (2002), 'Heterogeneous beliefs, risk and learning in a simple asset pricing model', Computational Economics 19, 95-132.

Chiarella, C. and He, X. (2003), 'Heterogeneous beliefs, risk and learning in a simple asset pricing model with a market maker', Macroeconomic Dynamics 7, 503-536.

Chiarella, C., He, X. and Wang, D. (2004), Asset price dynamics with time-varying second moment, Technical report, University of Technology, Sydney.

Dacorogna, M., Muller, U., Jost, C., Pictet, O., Olsen, R. and Ward, J. (1995), 'Heterogeneous real-time trading strategies in the foreign exchange market', European Journal of Finance 1, 383-403.

Day, R. and Huang, W. (1990), 'Bulls, bears and market sheep', Journal of Economic Behavior and Organization 14, 299-329.

Ding, Z., Granger, C. and Engle, R. (1993), 'A long memory property of stock market returns and a new model', Journal of Empirical Finance 1, 83-106.

Farmer, J. D. and Joshi, S. (2002), 'The price dynamics of common trading strategies', Journal of Economic Behavior and Organization 49, 149-171.

Gaunersdorfer, A. (2000), 'Endogenous fluctuations in a simple asset pricing model with heterogeneous agents', Journal of Economic Dynamics and Control 24, 799-831.

Gaunersdorfer, A., Hommes, C. and Wagener, F. (2003), Bifurcation routes to volatility clustering under evolutionary learning, Technical Report 03-03, CeNDEF, University of Amsterdam. Working Paper.

He, X. (2003), Asset pricing, volatility and market behaviour-a market fraction approach, Technical Report 95, Quantitative Finance Research Center, University of Techonology, Sydney. 
Hommes, C. (2001), 'Financial markets as nonlinear adaptive evolutionary systems', Quantitative Finance 1, 149-167.

Hommes, C. (2002), 'Modeling the stylized facts in finance through simple nonlinear adaptive systems', Proceedings of National Academy of Science of the United States of America 99, 7221-7228.

Kirman, A. (1992), 'Whom or what does the representative agent represent?', Journal of Economic Perspectives 6, 117-136.

LeBaron, B., Arthur, W. and Palmer, R. (1999), 'Time series properties of an artifical stock market', Journal of Economic Dynamics and Control 23, 1487-1516.

Lux, T. (1995), 'Herd behaviour, bubbles and crashes', Economic Journal 105, 881-896.

Lux, T. (1997), 'Time variation of second moments from a noise trader/infection model', Journal of Economic Dynamics and Control 22, 1-38.

Lux, T. (1998), 'The socio-economic dynamics of speculative markets: Interacting agents, chaos, and the fat tails of return distributions', Journal of Economic Behavior and Organization 33, 143-165.

Lux, T. and Marchesi, M. (1999), 'Scaling and criticality in a stochastic multi-agent model of a financial market', Nature 397(11), 498-500.

Manski, C. and McFadden, D. (1981), Structural Analysis of Discrete Data with Econometric Applications, MIT Press.

Pagan, A. (1996), 'The econometrics of financial markets', Journal of Empirical Finance 3, 15-102. 